\title{
Defining indications for selective chest radiography in the first 24 hours after cardiac surgery
}

\author{
Martijn Tolsma, MD, ${ }^{\mathrm{a}}$ Tom A. Rijpstra, MD, ${ }^{\mathrm{b}}$ Peter M. J. Rosseel, MD, ${ }^{\mathrm{b}}$ Thierry V. Scohy, MD, PhD, \\ Mohamed Bentala, MD, ${ }^{\mathrm{c}}$ Paul G. H. Mulder, MD, PhD, ${ }^{\mathrm{d}}$ and Nardo J. M. van der Meer, MD, PhD ${ }^{\mathrm{b}, \mathrm{e}}$
}

\section{ABSTRACT}

Objective: In the intensive-care unit (ICU), chest radiographs (CXRs) are frequently obtained routinely for postoperative cardiac surgery patients, despite the fact that the efficacy of routine CXRs is known to be low. We investigated the efficacy and safety of CXRs performed after cardiac surgery for specified indications only.

Methods: In this observational cohort study, we prospectively included all patients who underwent conventional major cardiac surgery by median sternotomy in the year 2012. On-demand CXRs could be obtained during the first postoperative period for specified indications only. A routine control CXR was performed on the morning of the first postoperative day for all patients who had not undergone a CXR before that time. The diagnostic and therapeutic efficacy values were calculated for all CXRs. Differences were tested using Fisher's exact test or $\chi^{2}$ analysis.

Results: A total of 1102 consecutive cardiac surgery patients were included in this study. The diagnostic efficacy of CXRs for major abnormalities was higher for the postoperative on-demand CXRs $(\mathrm{n}=301 ; 27 \%)$ than for the routine CXRs taken the morning after surgery $(\mathrm{n}=801 ; 73 \%)(6.6 \%$ vs $2.7 \%, P=.004)$. The therapeutic efficacy was higher for the on-demand CXRs, whereas the need for intervention after the next-morning, routine CXRs was limited to 5 patients $(4.0 \%$ vs $0.6 \%, P<.001)$. None of these patients experienced a major adverse event.

Conclusions: Defining clear indications for selective CXRs after cardiac surgery is effective and seems to be safe. This approach may significantly reduce the total number of CXRs performed, and will increase their efficacy. (J Thorac Cardiovasc Surg 2015;150:225-9)

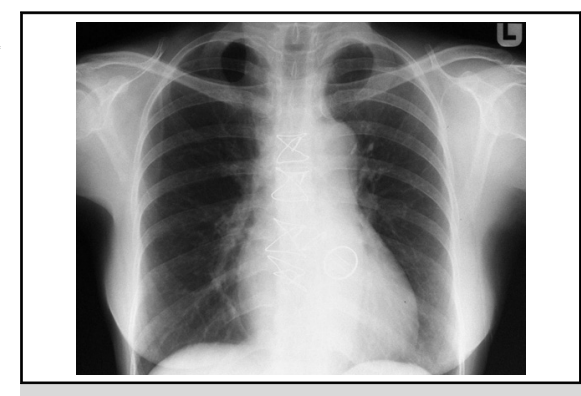

A normal chest radiograph after cardiac surgery.

\section{Central Message}

Defining clear indications for selective chest radiography after cardiac surgery is efficient and seems to be safe.

\section{Perspective}

Performing routine chest radiographs (CXRs) after cardiac surgery is a common worldwide practice, despite the fact that the efficacy of these radiographs is known to be low. In the context of creating optimal and cost-efficient postoperative care, our results show that selective postoperative CXR use is safe and (cost) efficient. Immediate postoperative CXR use was reduced by $73 \%$ in our study.

See Editorial Commentary page 230.
Chest radiographs (CXRs) are obtained frequently and routinely for intensive-care unit (ICU) patients, on a daily basis, and after surgery and certain other procedures. Multiple investigators have studied the clinical value of routine CXRs after central venous catheterization, endotracheal intubation, and chest tube placement or removal. ${ }^{1-12}$

\footnotetext{
From the ${ }^{\mathrm{a} D e p a r t m e n t}$ of Anesthesiology and Intensive Care, Isala Clinics, Zwolle; ${ }^{\mathrm{b}}$ Department of Anesthesiology and Intensive Care, ${ }^{\mathrm{c}}$ Department of Cardiothoracic Surgery, ${ }^{\mathrm{d}}$ Amphia Academy, Amphia Hospital, Breda, Oosterhout, and Etten-Leur;

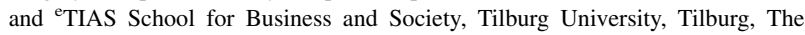
Netherlands.

Financed by departmental funding only. There was no external funding source.

Received for publication Feb 3, 2015; revisions received April 6, 2015; accepted for publication April 11, 2015; available ahead of print May 22, 2015.

Address for reprints: Martijn Tolsma, MD, Department of Anesthesiology \& Intensive Care, Isala Clinics, Dokter van Heesweg 2, 8025 AB Zwolle, The Netherlands (E-mail: m.tolsma@isala.nl).

0022-5223/\$36.00

Copyright (c) 2015 by The American Association for Thoracic Surgery http://dx.doi.org/10.1016/j.jtcvs.2015.04.026
}

Others have studied the value of daily routine CXRs in a mixed ICU population, or in mechanically ventilated patients only. ${ }^{13-21}$ The diagnostic and therapeutic efficacy of all these routine CXRs has been reported to be low. ${ }^{1-3,6-9,11,13,14,16-19,21}$

Investigators comparing a routine CXR strategy with an on-demand CXR strategy were not able to show any difference in outcome measures. ${ }^{22-28}$ Although those studies indicated that a more restrictive CXR strategy should be safe, a more recent meta-analysis by Ganapathy and colleagues ${ }^{26}$ stated that, in those studies, the confidence intervals were wide, and the study populations were small. In addition, they asserted that the potential harm and missed findings were not assessed rigorously enough. ${ }^{26}$ Meanwhile, the discussion regarding specific indications for CXRs in critically ill patients and the safety of abandoning routine CXRs is still ongoing.

In agreement with the results of studies on this topic in a general ICU population, the clinical value of routine CXRs 


\section{Abbreviations and Acronyms \\ $\mathrm{CABG}=$ coronary artery bypass grafting \\ $\mathrm{CXR}=$ chest radiograph \\ ICU = intensive-care unit}

after cardiac surgery is additionally known to be low. ${ }^{29-32}$ Despite these findings, our recent web survey of Dutch intensivists revealed that the strategy of daily routine CXRs is a rare practice nowadays, with the exception of routine CXRs for postoperative cardiac surgery patients. ${ }^{33}$ Abandoning routine CXRs after cardiac surgery may be safe only when patients at risk are identified and certain indications for CXRs are stated.

In 2011, we changed the protocol for CXRs after cardiac surgery in our department. Our former policy was to obtain routine CXRs for all postoperative cardiac surgery patients: at the moment of ICU arrival; on the morning of the first postoperative day; and after chest tube removal. We previously reported that the clinical value of CXRs using this strategy and the number of subsequent interventions were low. ${ }^{32}$ With the new protocol, a CXR in the direct postoperative period is performed only for certain indications. A routine CXR on the first postoperative morning is still performed for all patients, unless an on-demand CXR has been performed shortly before.

Our aim was to study the diagnostic and therapeutic efficacy of this new CXR strategy, performing CXRs after cardiac surgery for specified indications only. Our hypothesis was that the diagnostic efficacy of these postoperative CXRs, performed on a specified indication, would be higher. In addition, we expected that the diagnostic efficacy of the routine control CXRs, taken the morning after surgery for patients who did not meet any special indications, would be low.

\section{METHODS}

The study protocol was approved by the local ethics review board (at Amphia Hospital), and the need for written informed consent was waived because no interventions were conducted with the patients, apart from those that were part of the usual and current local practice. This prospective, observational, single-center study was performed in a tertiary center, 24bed, closed-format ICU that admits medical, surgical, and cardiac surgery patients. The medical staff of this ICU consisted of 12 intensivists and 8 ICU residents. All patient data were collected anonymously.

We prospectively included all consecutive patients who underwent conventional major cardiac surgery by median sternotomy in the year 2012. All patients were admitted to the ICU directly after surgery. According to our new strategy, a direct postoperative CXR upon ICU arrival was performed routinely for only certain specified indications (Table 1). These indications were chosen to confirm the correct positioning of the intra-aortic balloon pump and to rule out a pneumothorax or hemothorax after difficult central venous catheterization. Furthermore, a CXR could be obtained throughout the first postoperative period, according to other specified indications (Table 1) determined by an ICU physician, after an assessment that included interpretation of the patient's vital parameters, the results of an arterial blood gas sample, and auscultation of the heart and lungs. For all patients who did not undergo a CXR before the morning of the first postoperative day, a routine control CXR was performed at that time.

Demographic data and perioperative characteristics were collected for all patients. The mean age of patients and the mean duration of the ICU stays were calculated. All CXRs were assessed by both a radiologist and an ICU physician. The CXR findings were classified according to the overview presented in Table 2 and were divided into minor and major findings. Only new findings were analyzed, and abnormalities that were already present on a preoperative CXR were not taken into consideration for this study.

All CXR abnormalities were noted and categorized. An additional note was made if a major abnormality led to a subsequent intervention. Possible interventions were: chest tube placement, repositioning of invasive devices, diuretic therapy, echocardiography, and reoperation. The total numbers and fractions of CXRs that showed any findings, minor findings only, major findings, or findings that led to a subsequent intervention were calculated. The diagnostic efficacy (the number of abnormalities divided by the total number of CXRs) and therapeutic efficacy (the number of interventions based on CXR abnormalities, divided by the total number of CXRs) were calculated. All major findings were noted separately, whether or not they led to an intervention.

The data analysis was performed using IBM SPSS Statistics, version 21.0 for Windows (SPSS Inc, Chicago, Ill). Differences in the percentages of findings and interventions between the on-demand CXRs, versus the routine CXRs on the first postoperative morning, were tested using Fisher's exact test, or $\chi^{2}$ analysis when appropriate. To account for multiple testing, more-stringent criteria were used than the usual .05 significance level. A $P$ value of $<.01$ was considered to denote a statistically significant difference by intervention or finding.

\section{RESULTS}

Table 3 shows the baseline characteristics of the study population. A total of 1102 consecutive cardiac surgery patients were included in the study. Most patients $(73 \%)$ were men, and the mean patient age was $69 \pm 9$ years. The mean length of ICU stay was 2.0 nights. Most patients underwent coronary artery bypass grafting $(\mathrm{CABG})$, valve surgery, or a combination of the 2 .

For 301 patients $(27 \%)$, a CXR was performed on demand, at ICU admission, or at some point during the first postoperative period. The remaining 801 patients $(73 \%)$ had a routine control CXR taken on the morning of the first postoperative day. Table 4 shows the number and type of CXRs specified per procedure. Notably, the routine control CXR group consisted of almost two thirds CABG patients $(65 \%)$, because $79 \%$ of the CABG patients did not meet the criteria for an on-demand CXR before the next morning.

The values for various CXR findings are presented in Table 5. All CXRs had a comparable diagnostic efficacy for minor abnormalities, of approximately $45 \%$ $(P=.22)$. The diagnostic efficacy for major abnormalities was clearly higher for the on-demand CXR group than for the group who had routine CXRs the next morning $(6.6 \%$ vs $2.7 \%)(P=.004)$. The therapeutic efficacy was $4.0 \%$ for the on-demand CXRs, whereas the routine CXRs had a therapeutic efficacy of only $0.6 \%(P<.0005)$.

Table 6 shows an overview of all the major abnormalities found, and the frequency of subsequent diagnostic or 
TABLE 1. Established indications for an on-demand chest radiograph

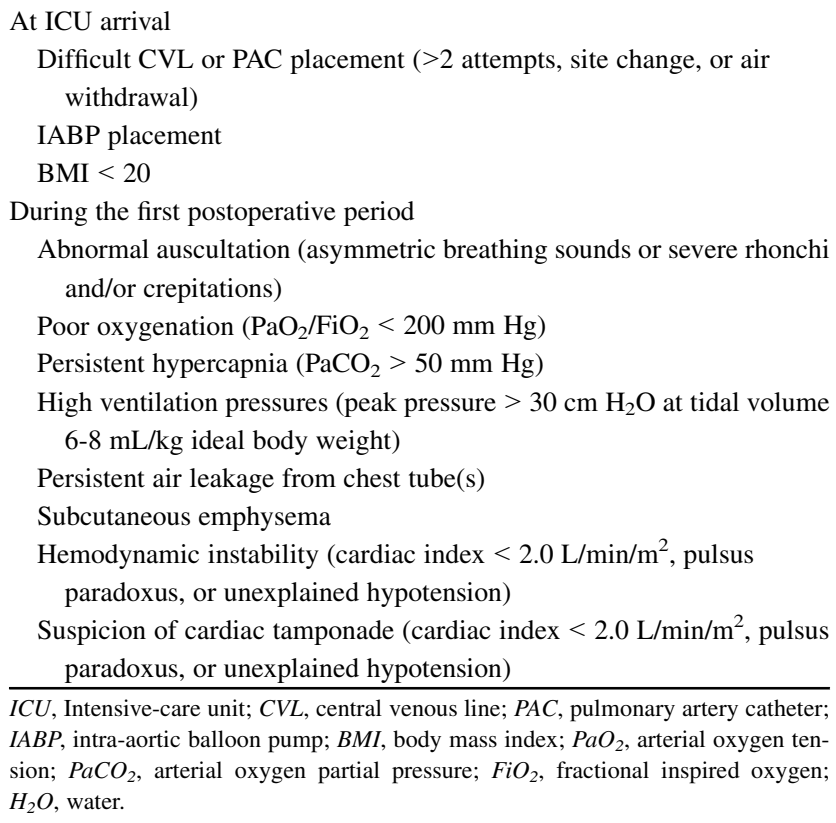

During the first postoperative period

Abnormal auscultation (asymmetric breathing sounds or severe rhonchi and/or crepitations)

Poor oxygenation $\left(\mathrm{PaO}_{2} / \mathrm{FiO}_{2}<200 \mathrm{~mm} \mathrm{Hg}\right)$

Persistent hypercapnia $\left(\mathrm{PaCO}_{2}>50 \mathrm{~mm} \mathrm{Hg}\right)$

High ventilation pressures (peak pressure $>30 \mathrm{~cm} \mathrm{H}_{2} \mathrm{O}$ at tidal volume 6-8 $\mathrm{mL} / \mathrm{kg}$ ideal body weight)

Persistent air leakage from chest tube(s)

Subcutaneous emphysema

Hemodynamic instability (cardiac index $<2.0 \mathrm{~L} / \mathrm{min} / \mathrm{m}^{2}$, pulsus paradoxus, or unexplained hypotension)

Suspicion of cardiac tamponade (cardiac index $<2.0 \mathrm{~L} / \mathrm{min} / \mathrm{m}^{2}$, pulsus paradoxus, or unexplained hypotension)

$I C U$, Intensive-care unit; $C V L$, central venous line; $P A C$, pulmonary artery catheter; $I A B P$, intra-aortic balloon pump; $B M I$, body mass index; $\mathrm{PaO}_{2}$, arterial oxygen tension; $\mathrm{PaCO}_{2}$, arterial oxygen partial pressure; $\mathrm{FiO}_{2}$, fractional inspired oxygen; $\mathrm{H}_{2} \mathrm{O}$, water.

therapeutic interventions. For the on-demand CXRs, the most frequent reasons for an intervention were: a hemothorax $(\mathrm{n}=3)$; a widened mediastinum $(\mathrm{n}=3)$; malposition of invasive devices $(\mathrm{n}=3)$; or a pneumothorax $(\mathrm{n}=2)$. Common interventions based on the CXR results were chest tube placement, reoperation, repositioning of an invasive device, and echocardiography. Only 5 of the 23 major abnormalities found on the routine CXRs, taken on the first postoperative morning, required an intervention. In 4 patients, a chest tube was placed because of a pneumothorax $(\mathrm{n}=3)$ or large pleural effusion $(\mathrm{n}=1)$. In 1 patient, echocardiography was performed after the presence of a widened mediastinum was detected on the routine CXR. Only a small amount of pericardial effusion was found in this case, for which no intervention was

TABLE 2. Classification of radiologic findings

\begin{tabular}{ll}
\hline \multicolumn{1}{c}{ Minor findings } & \multicolumn{1}{c}{ Major findings } \\
\hline Little pleural effusion & Severe pleural effusion \\
Small atelectasis & Large atelectasis \\
Little pulmonary congestion & Severe pulmonary congestion \\
Small consolidation & Large consolidation \\
& Malposition of invasive devices \\
& Widened mediastinum \\
& Large subcutaneous emphysema \\
& Hemothorax \\
& Pneumothorax \\
& Pneumomediastinum \\
& Pneumopericardium \\
& Free air under diaphragm \\
\hline
\end{tabular}

Minor findings are defined as involvement of $<1$ lobe, and/or judged to be a "normal postoperative finding." Major findings are defined as involvement of $\geq 1$ lobe, and/or judged to be a "non-normal postoperative finding."
TABLE 3. Baseline data of the study population and procedures

\begin{tabular}{lc}
\hline Characteristics and procedures & n (\%) \\
\hline Total number & $1102(100)$ \\
Gender, male & $809(73)$ \\
Age (y), mean \pm SD & $69 \pm 9$ \\
Length of ICU stay (d), mean (range) & $2.0(1-66)$ \\
$\quad$ Length of ICU stay, 1 d & $847(77)$ \\
Procedures & \\
CABG & $655(59)$ \\
CABG with valve surgery & $177(16)$ \\
CABG with rhythm surgery & $21(1.9)$ \\
Valve surgery & $140(13)$ \\
Valve surgery with aortic surgery & $42(3.8)$ \\
Valve surgery with rhythm surgery & $32(2.9)$ \\
Aortic surgery & $24(2.2)$ \\
Other surgery & $11(1.0)$ \\
\hline
\end{tabular}

Values are $\mathrm{n}(\%)$, unless otherwise indicated. $S D$, Standard deviation; $I C U$, intensivecare unit; $C A B G$, coronary artery bypass grafting.

necessary. These complications had no effect on ICU length of stay. Four patients left the ICU on the same day, and the fifth patient was kept 2 days longer for other reasons. All 5 patients were discharged from the ICU, and from the hospital, in good condition.

\section{DISCUSSION}

Our results show that the therapeutic and diagnostic efficacy of on-demand CXRs that are performed directly or shortly after cardiac surgery is clearly higher when specific indications are stated. The efficacy of a routine CXR taken on the first postoperative morning, for patients without a prior direct indication for a CXR, was very low.

The high diagnostic efficacy for minor findings $(45 \%)$ is in agreement with the findings of previous studies ${ }^{21}$; and similarly, a higher diagnostic efficacy was found for major findings $(7 \%$ compared with $3 \%)$ when CXRs were obtained on demand, rather than routinely. ${ }^{17,18,21}$ The generally low therapeutic efficacy of CXRs that we found is in agreement with previous findings as well. ${ }^{16-18,21}$ However, more importantly, the therapeutic value of our

TABLE 4. First obtained chest radiographs specified per procedure

\begin{tabular}{lrc}
\hline \multicolumn{1}{c}{ Procedure } & On-demand & $\begin{array}{c}\text { Morning } \\
\text { after surgery }\end{array}$ \\
\hline CABG $(\mathrm{n}=655)$ & $137(21)$ & $518(79)$ \\
CABG and valve surgery $(\mathrm{n}=177)$ & $66(37)$ & $111(63)$ \\
CABG and rhythm surgery $(\mathrm{n}=21)$ & $9(43)$ & $12(57)$ \\
Valve surgery $(\mathrm{n}=140)$ & $48(34)$ & $92(66)$ \\
Valve and aortic surgery $(\mathrm{n}=42)$ & $16(38)$ & $26(62)$ \\
Valve and rhythm surgery $(\mathrm{n}=32)$ & $8(25)$ & $24(75)$ \\
Aortic surgery $(\mathrm{n}=24)$ & $12(50)$ & $12(50)$ \\
Other surgery $(\mathrm{n}=11)$ & $5(45)$ & $6(55)$ \\
Total $(\mathrm{n}=1102)$ & $301(27)$ & $801(73)$ \\
\hline Values are $(\%)$ with $\%=$ row proportion
\end{tabular}


TABLE 5. Chest radiograph findings and subsequent interventions

\begin{tabular}{|c|c|c|c|c|}
\hline Findings and & $\begin{array}{c}\text { On-demand } \\
\text { CXR } \\
\end{array}$ & $\begin{array}{l}\text { CXR morning } \\
\text { after surgery }\end{array}$ & $\frac{\text { Total }}{2-110 ?}$ & $P$ \\
\hline & $\mathbf{n}=\mathbf{3 0 1}$ & $\mathrm{n}=\mathbf{8 0 1}$ & $\mathrm{n}=1102$ & \\
\hline Any finding & $147(49)$ & 393 (49) & $540(49)$ & 1.00 \\
\hline Minor findings only & $127(42)$ & $371(46)$ & $498(45)$ & .22 \\
\hline Major findings & $20(6.6)$ & $22(2.7)$ & $42(3.8)$ & .004 \\
\hline $\begin{array}{l}\text { Subsequent } \\
\text { interventions }\end{array}$ & $12(4.0)$ & $5(0.6)$ & $17(1.5)$ & $<.001$ \\
\hline
\end{tabular}

routine control CXR taken on the first postoperative morning $(0.6 \%)$ was far lower than reported in previous studies, indicating that the number of important findings missed by the on-demand CXRs was minimal.

According to our results, the practice of taking a routine CXR directly after cardiac surgery can be safely discontinued, as long as clear indications are stated for when patients need a direct postoperative CXR at ICU arrival, or an on-demand CXR throughout the first postoperative period. This strategy will significantly reduce the total number of CXRs that are performed for cardiac surgery patients. In our department, the number of immediate postoperative CXRs was reduced by $73 \%$ (801 CXRs in 1 year, with a total cost savings of approximately $\$ 40,000)$. The control CXRs taken on the first postoperative morning indicated that only a minimal number of important findings were missed using this strategy, and that an intervention after this type of routine CXR was rare ( $0.6 \%$ of patients). The findings that were revealed on these CXRs are unlikely to have caused any obvious clinical problems in the first postoperative period; thus, the delay in treatment until the next morning, as a result of not

TABLE 6. Specific major findings and number of interventions

\begin{tabular}{|c|c|c|c|c|}
\hline \multirow[b]{2}{*}{ Finding } & \multicolumn{2}{|c|}{ On-demand CXR } & \multicolumn{2}{|c|}{$\begin{array}{l}\text { CXR morning } \\
\text { after surgery }\end{array}$} \\
\hline & Found & Intervention & Found & Intervention \\
\hline Severe pleural effusion & 1 & 1 & 1 & 1 \\
\hline Large atelectasis & 1 & 1 & 1 & 0 \\
\hline $\begin{array}{l}\text { Severe pulmonary } \\
\text { congestion }\end{array}$ & 0 & 0 & 1 & 0 \\
\hline $\begin{array}{l}\text { Malposition invasive } \\
\text { devices }\end{array}$ & 6 & 3 & 4 & 0 \\
\hline Widened mediastinum & 7 & 3 & 6 & 1 \\
\hline Hemothorax & 3 & 3 & 0 & 0 \\
\hline Pneumothorax & 2 & 2 & 8 & 3 \\
\hline Pneumopericardium & 0 & 0 & 2 & 0 \\
\hline Free air under diaphragm & 1 & 0 & 0 & 0 \\
\hline Total & 21 & 13 & 23 & 5 \\
\hline
\end{tabular}

The total number of major findings and interventions may be higher in this table than in previous tables because some CXRs had $>1$ major finding. $C X R$, Chest radiograph. performing a routine CXR at ICU arrival, will probably not harm these patients. All 5 patients were discharged from the ICU within a short period, and no major adverse events occurred.

Furthermore, if correct indications are stated, the relevance of performing a routine CXR at all may be questionable; however, not doing so may conflict with our need for reassurance of the safety of our patients when they are transferred to the ward. Previously, we additionally showed a poor correlation between physical examination and CXR findings, ${ }^{32,34}$ and the few major findings on the control CXRs taken on the first postoperative morning were not otherwise clinically identified.

We suggest performing a direct postoperative CXR when an intra-aortic balloon pump is placed, or when central venous catheterization is problematic. In addition, an on-demand CXR can be performed during the first postoperative period, when certain hemodynamic or respiratory problems are present. For now, performing a routine CXR on the first postoperative morning seems reasonable for patients who did not have a CXR previously in the postoperative period. More research on the safety of completely abandoning routine postoperative CXRs, eg, in CABG patients, is necessary. Bedside, chest ultrasound by ICU physicians may be a promising alternative..$^{34,35}$

Our study is limited by the fact that it was a single-center study and was performed according to an already existing protocol. In addition, we used an observational cohort study design without randomization or blinding. On the other hand, this design allowed us to evaluate so-called "missed findings," for the whole cohort, using a routine control CXR on the first postoperative morning. The study population was large, and clearly defined CXR indications were stated.

\section{CONCLUSIONS}

Defining clear indications for selective CXRs after cardiac surgery is effective and seems to be safe. This approach may significantly reduce the total number of CXRs performed, and will increase their efficacy.

\section{Conflict of Interest Statement}

Authors have nothing to disclose with regard to commercial support.

\footnotetext{
References

1. Lessnau KD. Is chest radiography necessary after uncomplicated insertion of a triple-lumen catheter in the right internal jugular vein, using the anterior approach? Chest. 2005;127:220-3.

2. Lucey B, Varghese JC, Haslam P, Lee MJ. Routine chest radiographs after central line insertion: mandatory postprocedural evaluation or unnecessary waste of resources? Cardiovasc Intervent Radiol. 1999;22:381-4.

3. Sanabria A, Henao C, Bonilla R, Castrillón C, Cruz H, Ramírez W, et al. Routine chest roentgenogram after central venous catheter insertion is not always necessary. Am J Surg. 2003;186:35-9.
} 
4. Abood GJ, Davis KA, Esposito TJ, Luchette FA, Gamelli RL. Comparison of routine chest radiograph versus clinician judgment to determine adequate central line placement in critically ill patients. J Trauma. 2007;63:50-6.

5. Brunel W, Coleman DL, Schwartz DE, Peper E, Cohen NH. Assessment of routine chest roentgenograms and the physical examination to confirm endotracheal tube position. Chest. 1989;96:1043-5.

6. Lotano R, Gerber D, Aseron C, Santarelli R, Pratter M. Utility of postintubation chest radiographs in the intensive care unit. Crit Care. 2000;4:50-3.

7. Whitehouse MR, Patel A, Morgan JA. The necessity of routine post-thoracostomy tube chest radiographs in post-operative thoracic surgery patients. Surgeon. 2009;7:79-81

8. Eisenberg RL, Khabbaz KR. Are chest radiographs routinely indicated after chest tube removal following cardiac surgery? Am J Roentgenol. 2011;197:122-4.

9. Khan T, Chawla G, Daniel R, Swamy M, Dimitri WR. Is routine chest X-ray following mediastinal drain removal after cardiac surgery useful? Eur $J$ Cardiothorac Surg. 2008;34:542-4.

10. McCormick JT, O'Mara MS, Papasavas PK, Caushaj PF. The use of routine chest X-ray films after chest tube removal in postoperative cardiac patients. Ann Thorac Surg. 2002;74:2161-4.

11. Palesty JA, McKelvey AA, Dudrick SJ. The efficacy of X-rays after chest tube removal. Am J Surg. 2000;179:13-6.

12. Sepehripour AH, Farid S, Shah R. Is routine chest radiography indicated following chest drain removal after cardiothoracic surgery? Interact Cardiovasc Thorac Surg. 2012;14:834-8.

13. Silverstein DS, Livingston DH, Elcavage J, Kovar L, Kelly KM. The utility of routine daily chest radiography in the surgical intensive care unit. J Trauma. 1993;35:643-6.

14. Fong Y, Whalen GF, Hariri RJ, Barie PS. Utility of routine chest radiographs in the surgical intensive care unit. A prospective study. Arch Surg. 1995; 130:764-8.

15. Brainsky A, Fletcher RH, Glick HA, Lanken PN, Williams SV, Kundel HL. Routine portable chest radiographs in the medical intensive care unit: effects and costs. Crit Care Med. 1997;25:801-5.

16. Graat ME, Choi G, Wolthuis EK, Korevaar JC, Spronk PE, Stoker J, et al. The clinical value of daily routine chest radiographs in a mixed medical-surgical intensive care unit is low. Crit Care. 2006;10:R11.

17. Graat ME, Kroner A, Spronk PE, Korevaar JC, Stoker J, Vroom MB, et al. Elimination of daily routine chest radiographs in a mixed medical-surgical intensive care unit. Intensive Care Med. 2007;33:639-44.

18. Hendrikse KA, Gratama JW, Hove W, Rommes JH, Schultz MJ, Spronk PE. Low value of routine chest radiographs in a mixed medical-surgical ICU. Chest. 2007; $132: 823-8$.

19. Kager LM, Kröner A, Binnekade JM, Gratama JW, Spronk PE, Stoker J, et al, Review of a large clinical series: the value of routinely obtained chest radiographs on admission to a mixed medical-surgical intensive care unit. J Intensive Care Med. 2010;25:227-32.

20. Bhagwanjee S, Muckart DJ. Routine daily chest radiography is not indicated for ventilated patients in a surgical ICU. Intensive Care Med. 1996;22:1335-8.
21. Clec'h C, Simon P, Hamdi A, Hamza L, Karoubi P, Fosse JP, et al. Are daily routine chest radiographs useful in critically ill, mechanically ventilated patients? A randomized study. Intensive Care Med. 2008;34:264-70.

22. Krinsley JS. Test-ordering strategy in the intensive care unit. J Intensive Care Med. 2003; 18:330-9.

23. Krivopal M, Shlobin OA, Schwartzstein RM. Utility of daily routine portable chest radiographs in mechanically ventilated patients in the medical ICU. Chest. 2003; $123: 1607-14$

24. Hejblum G, Chalumeau-Lemoine L, Ioos V, Boëlle PY, Salomon L, Simon T, et al. Comparison of routine and on-demand prescription of chest radiographs in mechanically ventilated adults: a multicentre, cluster-randomised, two-period crossover study. Lancet. 2009;374:1687-93.

25. Oba Y, Zaza T. Abandoning daily routine chest radiography in the intensive care unit: meta-analysis. Radiology. 2010;255:386-95.

26. Ganapathy A, Adhikari NK, Spiegelman J, Scales DC. Routine chest X-rays in intensive care units: a systematic review and meta-analysis. Crit Care. 2012;16:R68.

27. Kröner A, Binnekade JM, Graat ME, Vroom MB, Stoker J, Spronk PE, et al. Ondemand rather than daily-routine chest radiography prescription may change neither the number nor the impact of chest computed tomography and ultrasound studies in a multidisciplinary intensive care unit. Anesthesiology. 2008;108:40-5.

28. Mets O, Spronk PE, Binnekade J, Stoker J, de Mol BA, Schultz MJ. Elimination of daily routine chest radiographs does not change on-demand radiography practice in post-cardiothoracic surgery patients. J Thorac Cardiovasc Surg. 2007; 134:139-44.

29. Hornick PI, Harris P, Cousins C, Taylor KM, Keogh BE. Assessment of the value of the immediate postoperative chest radiograph after cardiac operation. Ann Thorac Surg. 1995;59:1150-4.

30. Karthik S, O'Regan DJ. An audit of follow-up chest radiography after coronary artery bypass graft. Clin Radiol. 2006;61:616-8.

31. Rao PS, Abid Q, Khan KJ, Meikle RJ, Natarajan KM, Morritt GN, et al Evaluation of routine postoperative chest X-rays in the management of the cardiac surgical patient. Eur J Cardiothorac Surg. 1997;12:724-9.

32. Tolsma M, Kröner A, van den Hombergh CL, Rosseel PM, Rijpstra TA, Dijkstra HA, et al. The clinical value of routine chest radiographs in the first 24 hours after cardiac surgery. Anesth Analg. 2011;112:139-42.

33. Tolsma M, Rijpstra TA, Schultz MJ, Mulder PG, van der Meer NJ. Significan changes in the practice of chest radiography in Dutch intensive care units: a web-based survey. Ann Intensive Care. 2014;4:10.

34. Vezzani A, Manca T, Brusasco C, Santori G, Valentino M, Nicolini F, et al Diagnostic value of chest ultrasound after cardiac surgery: a comparison with chest X-ray and auscultation. J Cardiothorac Vasc Anesth. 2014;28:1527-32.

35. Oks M, Cleven KL, Cardenas-Garcia J, Schaub JA, Koenig S, Cohen RI, et al The effect of point-of-care ultrasonography on imaging studies in the medical ICU: a comparative study. Chest. 2014;146:1574-7.

Key Words: Chest radiographs, cardiac surgery, postoperative care 